\title{
Cluster Head Energy Optimization in Wireless Sensor Networks
}

\author{
Shahab Tayeb*, Miresmaeil Mirnabibaboli and Shahram Latifi \\ Department of Electrical \& Computer Engineering, University of Nevada, \\ Las Vegas, NV, United States \\ *Corresponding Author: shahab.tayeb@unlv.edu
}

Received 24 October 2016; Accepted 20 November 2016; Publication 30 November 2016

\begin{abstract}
Wireless Sensor Networks (WSNs) consist of many sensor nodes which are vital to various applications in our daily lives. Optimizing energy usage is a key challenge for WSNs. Improving energy utilization can help with controlling traffic, saving energy and eventually, resulting in a better lifetime. In this paper, a modification of the Energy Efficient Credit-Based routing algorithm is proposed, which selects the most optimal Cluster Head based on the priority of relay nodes. Simulation results demonstrate that the proposed algorithm achieves a more efficient load-balancing, a better lifetime, and lower energy consumption, at the expense of slightly higher packet loss and lower data delivery rate. The results are compared with the commonly used Low-Energy Adaptive Clustering Hierarchy algorithm.
\end{abstract}

Keywords: Clustering, Energy Efficient Routing, Load-balancing.

\section{Introduction}

Wireless Sensor Network (WSN) is a distribution of autonomous sensors, which cooperatively monitor physical or environmental conditions, such as temperature, vibration, pressure, sound and so on. WSNs are used in many areas, including home automation, health monitoring or other healthcare

Journal of Software Networking, 137-162.

doi: 10.13052/jsn2445-9739.2016.008

(c) 2016 River Publishers. All rights reserved. 
applications, industrial process control and monitoring, etc. Applications of WSNs are expanding and the implementation of multifunctional and reliable WSNs is of utmost importance.

The detection process in WSNs mostly depends on sensor node's physical conditions and the solutions of detection problems are largely based on hardware rather than software. After detection, the node has to find whom and how to transfer the sensed data. After that, the turn passes to data transfer process. This process doesn't take much effort from sensor node due to the small size of desired data.

Low energy consumption is a critical task in WSNs, especially in sensor networks comprised of nodes that are considered lightweight with limited battery power. The most critical process in sensor networks is the routing because of high energy consumption, end-to-end delay, and control of packet overhead. Thus, it is required to have a routing mechanism for reducing energy consumption in sensor nodes and for increasing the network lifetime. The faster is the routing process, the longer is the sensor node lifetime and the less is the energy consumption. Hence, the development of efficient routing algorithms is a crucial task in WSN.

On one hand, low energy consumption is an important limitation in sensor networks, which are comprised of lightweight nodes with limited battery power. Hence, preserving the energy becomes a critical task in such networks. On the other hand, routing is a critical process in sensor networks due to concerns about energy consumption, end-to-end delay, and packet overhead. Thus, it is required to have a good routing mechanism in WSNs for reducing energy consumption in sensor nodes and for increasing the network lifetime. The process of setting up the routes during the initialization is influenced by energy considerations. Furthermore, load-balancing the resources evenhandedly prevents bottlenecks from forming and this is another challenging task $[1,2]$.

To increase the performance of WSN routing, multiple paths can be used concurrently. In coherent routing, the data is propagated after such processing as duplicate prevention, time-stamping, etc. The performance of routing protocols is linked to the architectural model and depends heavily on the implementation model. Design constraints might further impact the performance [3]. The routing protocol, especially with regard to route stability and the minimization of energy consumption, is significantly influenced by its data delivery model (Figure 1).

When it comes to routing in WSNs, energy consumption is not the only consideration. Such parameters as reliability, end-to-end delay, throughput, 


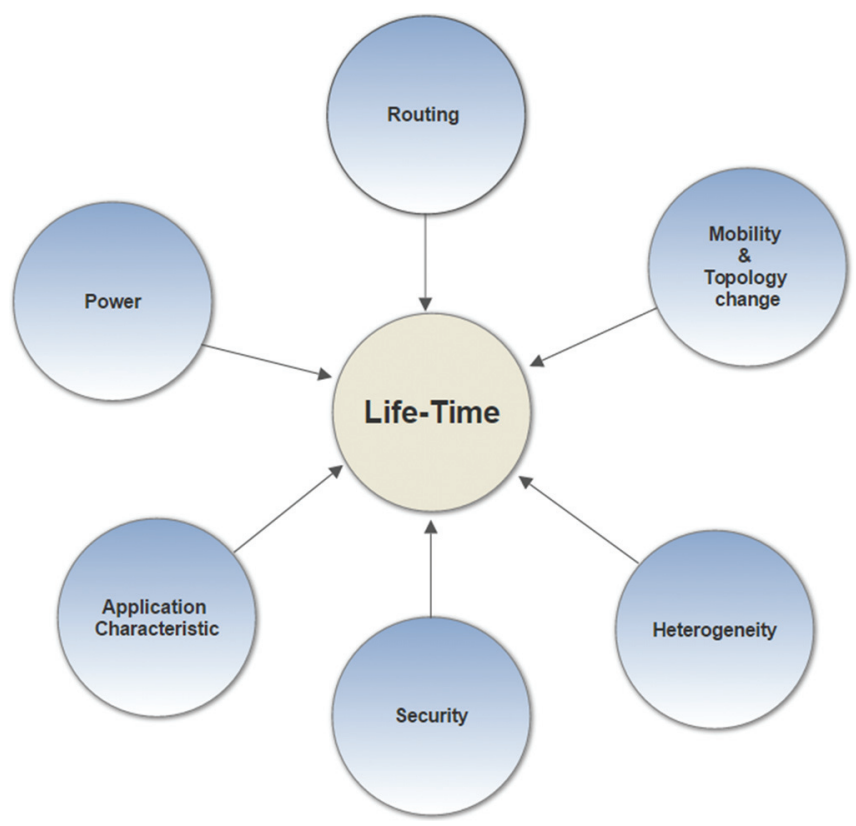

Figure 1 Illustration of parameters affecting routing.

or other QoS metrics are important when selecting the routing. These measures are directly related to bottleneck avoidance or to load-balance the energy consumption for increasing node and/or network performance and lifetime. Avoiding bottlenecks depends on the traffic load, which is in turn influenced by load balancing. Improper load-balancing will lead to unstable routes, which adds to the energy burden on sensors nodes and potentially deteriorates the network. Another consequence of improper load-balancing is partitioning the network into two or more segments. Energy consumption is one of the constraints and some literature [4] claim Dynamic Source Routing (DSR) protocol is more suitable in terms of small energy density.

The remainder of this paper is organized as follows. Section 2 discusses the related works on routing protocols and the issues associated with these algorithms in terms of load-balancing, bottleneck prediction, and congestion avoidance and management. In Section 3, the proposed algorithm is introduced, which incorporates load-balancing, congestion avoidance, and bottleneck avoidance. The performance and evaluation of our proposed algorithm, results and discussions are presented in Section 4. In Section 5, conclusion and future work, as well as recommendations, are presented. 


\section{Literature Review}

The routing protocol performance is a function of to the architectural model, for sensor networks have been considered different architectures and design constraints [4]. For route stability, energy and bandwidth are important optimization factors. There are different setups that utilize mobile sensors and the most part of network architectures sensor nodes are assumed as stationary. Node deployment affects the performance of the routing protocol and it is application dependent. The deployment can be either self-organizing or deterministic. In self-organizing systems, infrastructure is created in an ad hoc manner and the sensor nodes are scattered randomly. In deterministic situations, data is routed through pre-determined paths and the sensors are manually placed. The position of the cluster-head or the sink is also crucial in terms of energy efficiency and performance.

The process of setting up the routes during the creation of an infrastructure is influenced by energy considerations. If all nodes are in close proximity of the sink, direct routing would perform well enough. But in general multi-hop routing becomes unavoidable as sensors are scattered randomly over an area of interest. The routing protocol, especially with regard to route stability and the minimization of energy consumption, is highly influenced by the data delivery model. The sensor node in a sensor network can be associated with various functionalities. A node can be dedicated to a special function such as sensing, aggregation, relaying, depending on the application. As a result, the energy of node might quickly drain in case of the three functionalities engaging at the same time and it is a critical aspect in perspectives of node capability. To reduce the transmission in sensor nodes is using data aggregation when similar packets from multiple nodes are aggregated. For that purpose, sensor nodes are generating significant redundant data. In some sensor network applications, it is only required the success of messages delivery between a destination and a source. Other ones need even more assurance. The requirements of the real-time delivery and the maximization of network lifetime are:

a. Non-real time delivery: The message delivery assurance is indispensable for all routing protocols, which means that the protocol always has to find the route (if it really exists) between the communicating nodes.

b. Real-time delivery: Sometimes it is required that a message is delivered in a specified time [2], otherwise the message's information content is decreasing or the message becomes useless after time bound. Anyway, 
the main objective of these protocols is the network delay to complete control. These protocols' average-case performance may be evaluated by the time constraints and message delivery ratio measuring.

c. Network lifetime: This is crucial in networks, where the application has to run as long as possible. The metric which is used for determining the network lifetime is application dependent. In most protocols, it is assumed that every node is equally important and in such protocols as a metric is used either the time until the first node dies, or the nodes average energy consumption. But in case of nodes are not equally important, a reasonable metric can be the time till high-priority or the last nodes die.

The prediction process in WSNs mostly depends on sensor node's physical conditions. Prediction solutions are largely hardware-based. After prediction, the routing process takes place; followed by, the node finding the path for transferring the sensor data. After that, the route is selected and data is transferred. Due to the small volume of sensor-generated data, processing doesn't put a burden on the sensor node. Routing paths are established by one of the following methods: reactive, proactive, or hybrid [5, 6]. Proactive protocols compute and determine paths and save them in a table in the memory of each sensor, even before they are needed. Any changes should be propagated throughout the network. Updates to the routing table for each sensor requires large memory and resources because WSNs are comprised of thousands of sensors. In case of reactive protocols, routes are calculated only when they are looked up. Hybrid protocols combine characteristics of both reactive and proactive protocols [5].

Generally, WSN routing protocols are divided into three categories: hierarchical-based, location-based, and flat-based routing. In hierarchicalbased protocols, sensors perform different tasks. Scalability is the main sensor attribute in the design of such networks. Hierarchical-based routing operates in two modes. The first mode is used for selecting Cluster Heads $(\mathrm{CH})$ while the second is for routing and identifying a specific event. In locationbased routing, sensors' locations are used to route data. Moreover, location and position information are required to compute the distance between two given sensors. This way, the average energy consumption can be determined. Information about the location can be obtained using two techniques: one is to calculate the neighbor node, and the other is using GPS. In flat-based routing, all sensors share the same set of tasks in the network. Sensor selection for querying is difficult due to lack of global ID along with random extensions of nodes [7]. 
Routing protocols such as Directed-Diffusion (DD) routing [8], Sensor Protocol for Information via Negotiation (SPIN) [9, 10], Low Energy Adaptive Clustering Hierarchy (LEACH) [11], Gradient-based Routing (GBR) [12], Dynamic Source Routing (DSR) [13], and Power-Efficient Gathering in Sensor Information Systems (PEGASIS) [14] were introduced for efficient multi-hop routing in WSNs [15]. SPIN cannot guarantee 100 percent delivery of packets from a source node to the sink node. Moreover, SPIN needs a complete knowledge of the topology. DD is a data-centric protocol and it is mandatory for the sink node to create, transfer, and re-route the intermittent updates packets. The aim of designing DD was efficiency in energy consumption, resulting in an increase in network life expectancy. To reduce energy consumption, DD uses compression and processing of information within the network. However, it has limitation caused by the significant diffusion which results in a high level of overload. Furthermore, each node forms a gradient during the propagation toward all its neighbours. These gradients are paths used to route the packet. However, they provide limited information in the sense that each node is only capable of recognizing its immediate neighbour(s). As a result, DD is susceptible to bottlenecks, which causes inefficiency in energy consumption. Battery-Powered Sensor Nodes (BPSNs) can rarely meet design goals of long network lifetime and high reliability [16].

Directed Diffusion [8] is suitable for most applications but performs weakly for scenarios where there are many receivers and reference points. When receivers are related to one other, the data volume increases significantly. Gradient-based routing is a modified version of DD $[9,17]$. This solution uses such techniques as data aggregation and congestion management in order to balance the traffic uniformly, which helps in balancing the load on sensor nodes and thus, increases the network lifetime [17]. The LEACH routing algorithm is characterized as hierarchical and is designed to gather and receive data from and to the sink node, which essentially acts as a base station for ad-hoc networks $[18,19]$. PEGASIS and LEACH algorithms are similar. They both use a multi-hop algorithm for routing while a single sensor node is selected for forwarding to the sink node. Each node is a member of a chain for forwarding packets, resulting in a decrease in overhead. Using dynamic clustering in PEGASIS, performance increases three times.

\subsection{Classic Protocols for Improving Load-Balancing}

Clustering protocol is the commonly used algorithm focusing on lifetime, energy, and load balancing. Clustering offers scalability and location 
awareness while supporting mobility and data aggregation [19]. Data aggregation is combining data packets from multiple sensors in a single packet, using functions such as min, max, average, or duplicate removal. Data aggregation controls the load-bar which results in a decrease in the total number of packets.

In clustering algorithms, the load is balanced via dynamic selection of $\mathrm{CH}$ which provides good balancing of the energy of sensor nodes [20]. By the help of cluster rotation, $\mathrm{CH}$ transmits to all sensor nodes, resulting in a balanced consumption of energy throughout sensor lifetime. However, because of using multi-hop transition, $\mathrm{CH}$ located near the sink, have to transmit more traffic than other $\mathrm{CH}$ which creates such issues as bottleneck and congestion. As a result, the closest $\mathrm{CH}$ will be lost sooner than other $\mathrm{CH}$ [21]. Congestion avoidance, bottleneck prediction, and load-balancing are the key challenges in the WSN. Optimizing selection of sensor nodes helps with congestion management and facilitates load-balancing. For bottleneck prediction throughout network lifetime, sensors are controlled via monitoring buffer capacity and channel usage. On the other hand, congestion management and avoidance mechanisms can increase the performance of network and balance traffic load in multi-hop routing [18].

\subsection{Control Plane}

Reference [22] proposed an algorithm where the sensor node is aware of its own geographical location, using GPS, and is also aware of all its neighbours' locations. Additionally, the node is aware of the sink node. An enhanced version of this algorithm is proposed in Ref. [23], which utilizes buffer capacity and compares the node buffers. This facilitates congestion avoidance and prediction.

In Ref. [24], multi-path routing, i.e. load-balancing, is used in order to increase the throughput of the network. Moreover, the proposed algorithm is a hybrid method which performs congestion avoidance using traffic-aware routing. It also normalizes queue length and the depth of sink node from sensor nodes. In other words, it maintains a routing table in order to the least depth, or the shortest route.

Reference [25] proposed a fair cooperative routing method for heterogeneous overlapped WSN. To retain the total energy, this paper introduced an energy pool and used a cooperative packet-forwarding mechanism acting as an agent for fair cooperation. In Ref. [26], an application-driven algorithm was used based on energy-efficient node selection. This algorithm introduced 
an application-driven development which increases network lifetime and supports load-balancing via grouping sensor nodes running similar applications. Despite the increased delay, an improvement in load-balancing and lifetime is achieved. Reference [27] proposed a hybrid nature-inspired optimizer for WSN where they introduced three multi-objective models for the planning stage, namely, Load-Balanced Model, Interference Model, and Flow-Capacity Model. In Ref. [28], they presented a method based on the results of a two-year deployment. This included 455 wireless energy plug-load devices as well as seven load-balancing routers, which were implemented into a building for one year. Abdelhakim et al. [29] proposed an energy-efficient algorithm for timesensitive application using optimal topology design. Ren et al. [30] analysed network lifetime for BPSN and proposed an energy-based analytical model. Deva et al. [31] introduced deputy $\mathrm{CH}$ for alternate path selection, resulting in higher energy efficiency and throughput for WSNs.

\section{The Proposed Algorithm}

In Ref. [2], a clustering algorithm called Energy Efficient Credit-Based (EECB) is introduced, a clustering algorithm which uses candidate nodes for transmission between clusters. The relay node provides an optimum route for energy efficiency. The suggested method for an optimized routing selection with division of duties between nodes in the method of Directed-Diffusion, changes the flat routing into the hierarchical one. The change continues to achieve a suitable consumption of energy and ignorance of nodes which are not on the way of sink-source. This prevents a waste of energy. In fact, the transformations of flat routing into hierarchical routing causes a condition for the method of Directed-Diffusion, where we can insert different duties for nodes, based on practical policies, and divide the nodes, based on duties. At the beginning, the duty division takes place based on local clustering [2] and the nodes compete to be a cluster head. After a period, a node with the highest remaining energy than its geographical area nodes is selected as the cluster head node. If the reminder energy of the cluster head node is less than the threshold, it will enterprise a new cluster replacement and the competitor node with maximum reminder energy will be replaced by previous cluster head node. It means that the sensors with maximum reminder energy are selected as a cluster (Figure 2). This is like some sensors get very close locations. Thus, the sensor selects the wireless with the maximum reminder energy as the first cluster. This selection can be done based on Equation (1) of selection 


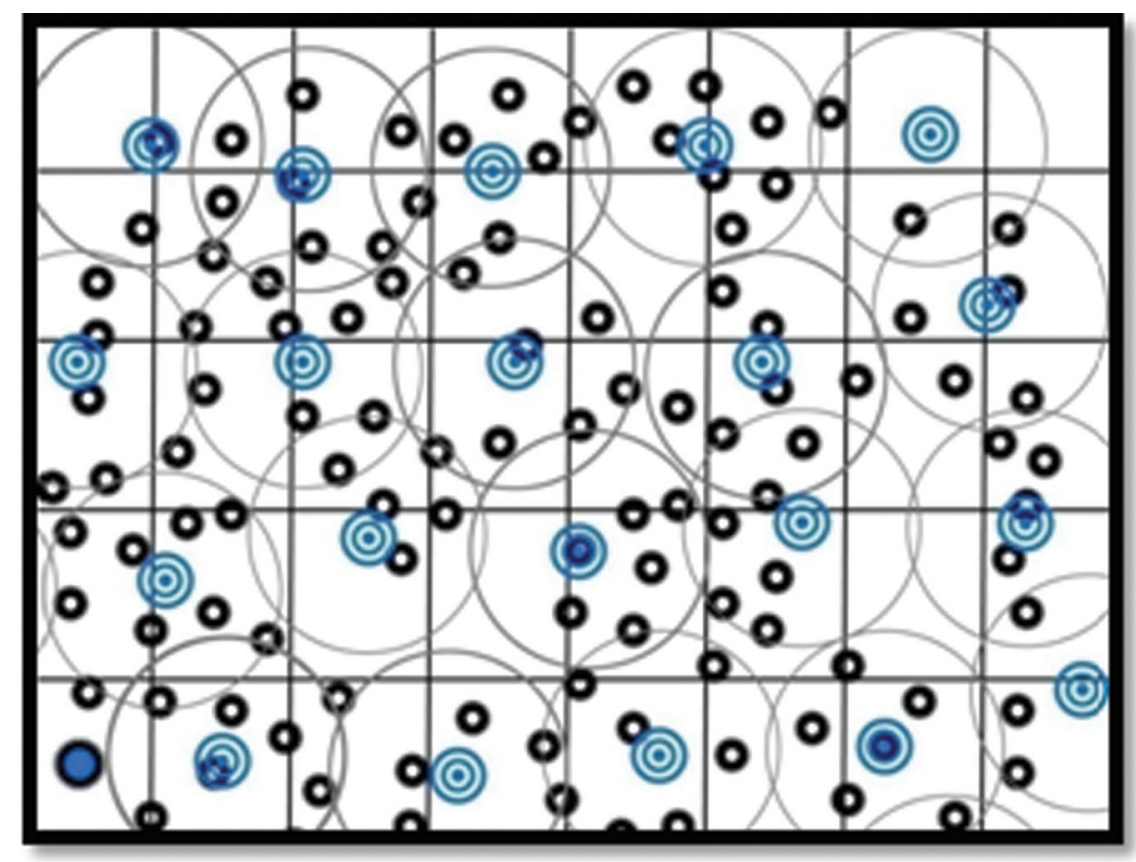

Figure 2 Illustration of arrangement and clustering of network nodes.

of alternative cluster head (Figure 2). Each wireless sensor with a maximum factor other than primary cluster head is selected as the alternative cluster head.

$$
\begin{aligned}
\mathrm{CH} \_\mathrm{R}_{F}= & \mathrm{E}_{N i}-\log _{10}\left(\mathrm{~d}_{N i \text { to primary } C H}\right) \\
& -\log _{10}\left(\mathrm{AVG}_{-} \mathrm{D}_{N i \text { to primary } C H}\right)
\end{aligned}
$$

where $\mathrm{E}_{N i}$ is the energy of the candidate node for replacement, $\mathrm{d}_{N i}$ to primary $\mathrm{CH}$ is the distance between an alternative node to the primary cluster head. AVG_D shows the delay between the alternative node and the cluster head.

When a node senses an event, a packet named event with a determined lifetime is created and it is sent to the cluster head. The cluster head node sends the discovered packet to the adequate relay node by use of a specified function, in order to send the adequate cluster head. The research will deal with this issue in the next parts of this dissertation; there is an important point in sending packet by cluster. In other words, if the sink node is in the sending limitation of cluster head radius or there is not any relay node, data will be sent to the sink node directly. Otherwise, the cluster head selects the node 
with the highest amount of route choice as the next hop. Figure 3 demonstrates how clustering takes place and Figure 4 proposes a flowchart for $\mathrm{CH}$ replacement.

In this part, we introduce some parameters for selecting the best relay node and after that, making a list of relay nodes based on priority. The priority of relay nodes includes the following parameters.

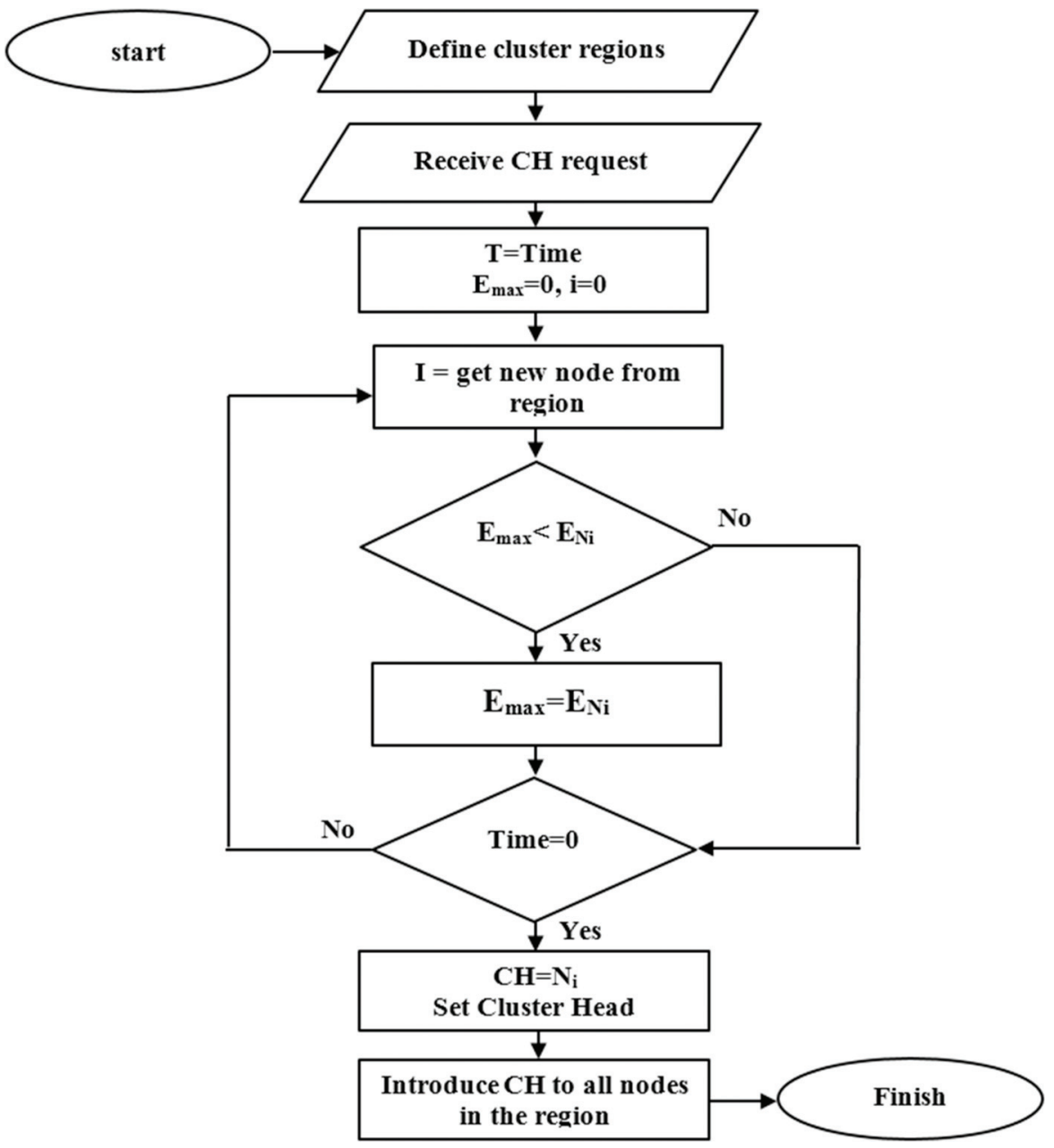

Figure 3 Flowchart of clustering. 


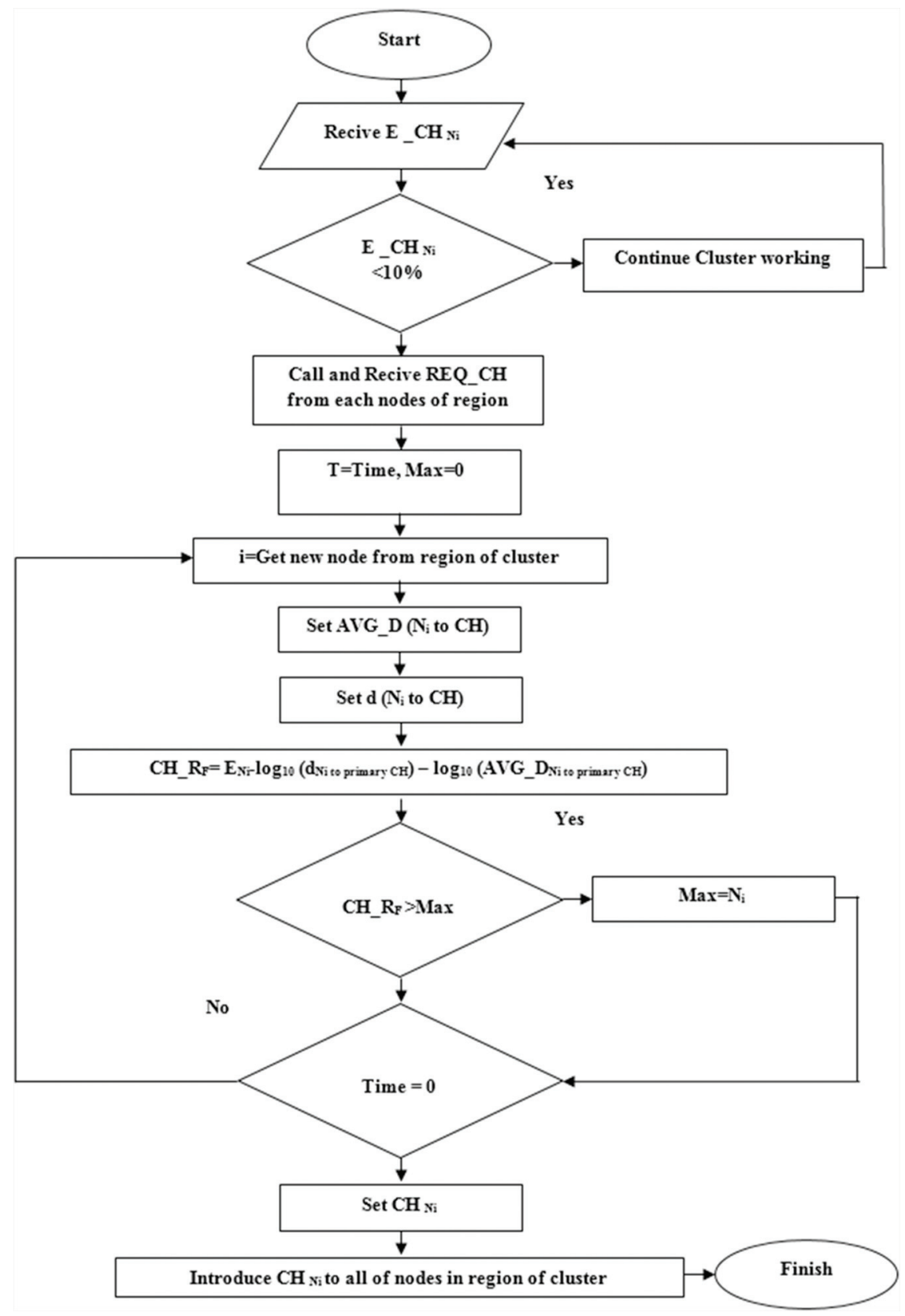

Figure 4 Cluster head replacement flowchart. 


\subsection{Candidate Relay Nodes $\left(\mathbf{C}_{N}\right)$}

Cluster Head $(\mathrm{CH})$ selects the best nodes for data transmission based on the estimate the send-radius. In this paper, Equation (2) is used to select the set of candidate nodes. Using Equation (2), the distance between the current node and the $\mathrm{CH}$ is estimated. It is then subtracted from the send-radius $\left(x_{s}\right)$. If the result is within the pre-defined threshold, the node is selected as a candidate relay node. The details are illustrated in Figure 5.

$$
C_{N}=\left\{C_{i}|\rightarrow|\left(d_{\text {Node } . \text { to . } C H}-x_{s}\right) \mid \leq \lambda\right\}
$$

where $C_{i}$ represents a given node.

\subsection{Feasibility Condition (FC)}

The distance from the candidate node to the sink node: The distance of the node $i$ to the sink is designated as $d_{N i}$. It should be noted that each node is aware of its own distances to the sink node. The relationship between the distance and mean energy consumption is reciprocal; i.e. a more distant node has a higher mean of energy consumption which causes more delay for establishing adjacency with the sink node. As it can be seen in Figure 6, node A is closer to the sink node, than node B. If B is selected for transmission, the routing

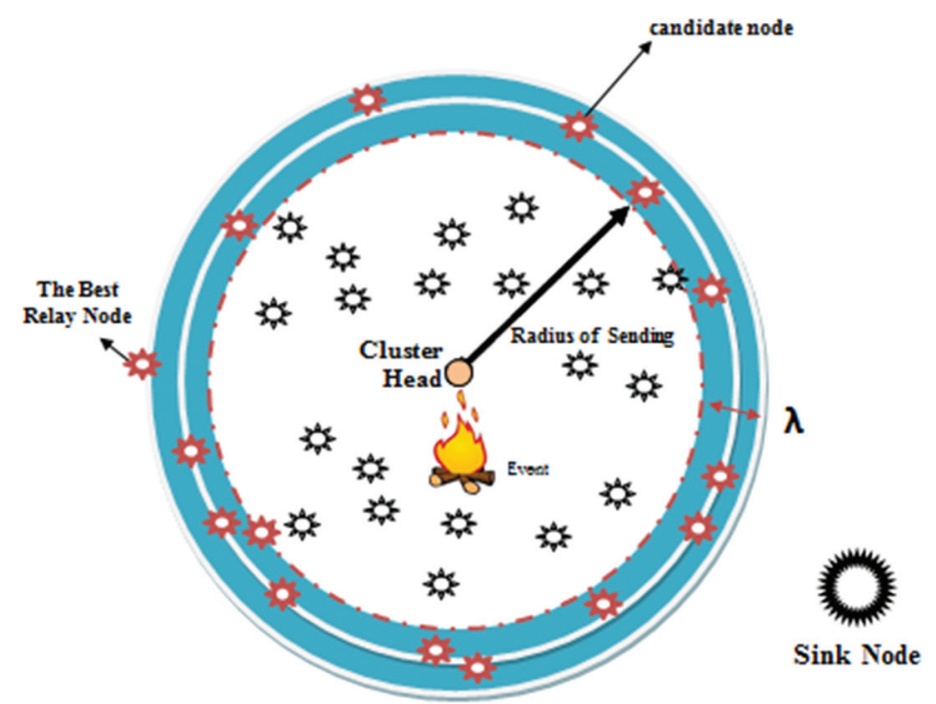

Figure 5 Illustration of node's sending radius and set of candidate nodes. 


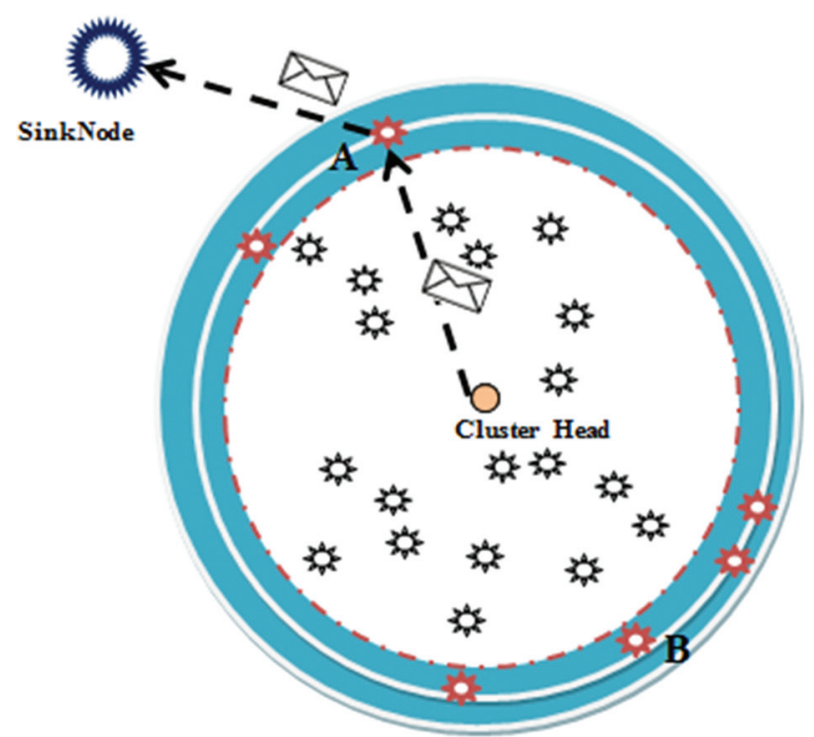

Figure 6 Effect of sending the distance to sink node.

map will change completely and the sink will receive the packet with a higher delay.

The distance of candidate node from the sender node: $d_{N i . t o . C H}$ is the distance of a given node to $\mathrm{CH}$.

The remainder energy in the candidate node: $E_{R}$ is the remainder of energy.

The amount of available buffer memory in the candidate node: the available space in the buffer memory is calculated using Equation (3).

$$
M_{F}=M_{t}-M_{e}
$$

where $M_{F}$ is the available buffer, $M_{t}$ is the total capacity of buffer and $M_{e}$ is the existing data in the buffer.

End-to-end delay: End-to-end delay is calculated using Equation (4).

$$
D_{\text {end-end }}=\left(D_{\text {trans }}+D_{\text {prop }}+D_{\text {proc }}\right)
$$

where $D_{\text {end-end }}$ is the end-to-end delay, $D_{\text {trans }}$ is the transmission delay whereas $D_{\text {prop }}$ and $D_{\text {proc }}$ are the propagation and process delay, respectively.

The ratio of failure/success: The ratio of failure to success in each time period is another parameter to recognize a suitable node. In this article, a self-variable memory for each sensor node is used, which is called $S_{l i}$. 
Each successful transmission results in a one-unit increase in the $S_{l i}$ amount. The ratio of success or failure can be calculated using Equation (5).

$$
S_{s}=\left(\frac{\sum S_{l i}}{\sum N_{i}}\right) \times 100
$$

Node selection is carried out using Equation (6). Figure 7 illustrates this offline process step-by-step:

$$
\begin{aligned}
F_{i}= & E_{R i}+\alpha_{1} \log d_{N i . t o \cdot \sin k}-\alpha_{2} \log d_{N i . t o . C H} \\
& -\alpha_{3} \log M_{F}-\alpha_{4} \log D_{\text {end.to.end }}-\alpha_{5} \log S_{s}
\end{aligned}
$$

$F_{\max }$ is the selected node and the route selection process depends on the choice of a relay node. After selection, $K_{i j}$ is formed where $i$ is the node number and $j$ is the cluster number. This process is repeated for every other cluster. Each relay node is selected from the set of candidate nodes. Sensor $R_{i}$ detects a destination object $O_{i}$ if it is one of the candidate relay nodes. Equation (7) shows the binary model of route selection.

$$
\text { Route }(\mathrm{s}) \leftarrow \begin{cases}1, & F\left(R_{i}, O_{i}\right) \leq r \\ 0, & F\left(R_{i}, O_{i}\right)>r\end{cases}
$$

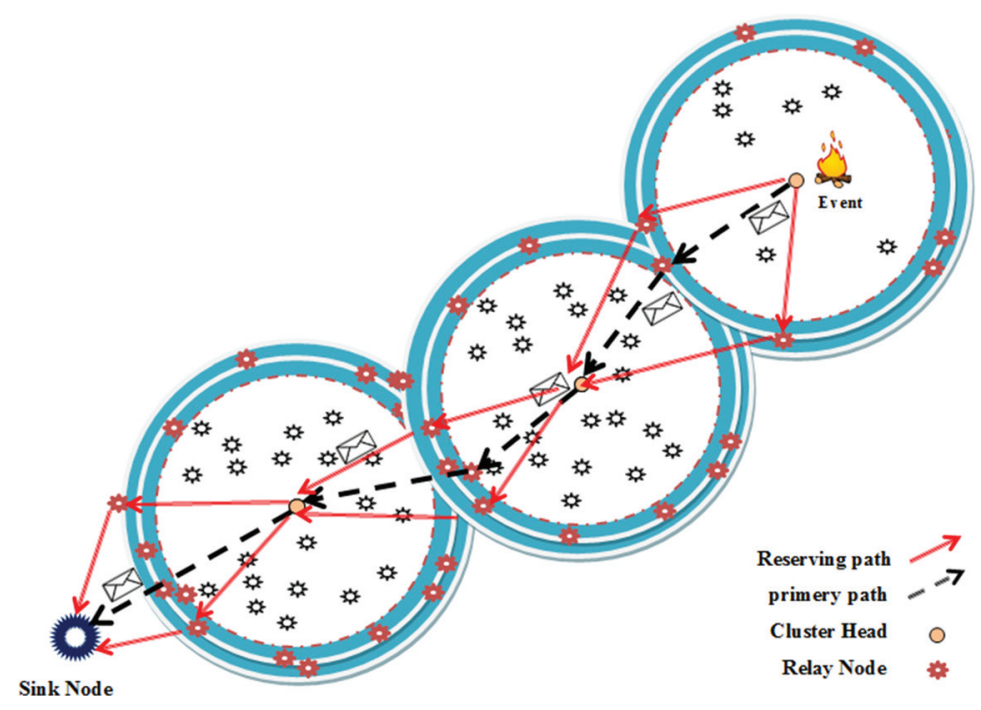

Figure 7 Routing map by clustering and selection of optimum relay node. 
where $F$ is the selection function for relay node $R_{i}$, the sensed target object $O_{j}$ and $r$ is the sending radius. When the target object is included, the route function(s) equals to 1 , otherwise is 0 .

\section{Experimental Results and Analysis}

To implement the proposed algorithm, MATLAB was used. 200 sensor nodes were stochastically distributed into $100 \mathrm{~m}^{2}$. After that, these nodes were divided into several clusters, illustrated by different colours. Then, routing map is established based on the LEACH algorithm (Figure 8). The best relay nodes are selected based on Equation (5). Figure 9 demonstrates the optimum path selection. Figure 10 represents the establishment of routing map using the proposed algorithm and Figure 11 optimizes the path selection, accordingly. In the simulations, five clusters were formed after random distributions of nodes. Three routes were found, which are used alternatively based on the Markov chain (Figures 11-13). Figure 12 performs route selection using Markov chain and Figure 13 chooses the optimum path from Figure 12. The gathered information is sending via wireless sensor network to monitor or controller that is called sink. The Sink can use the data and event locally from its environment, or it can resend data to other nodes or station from wireless network.

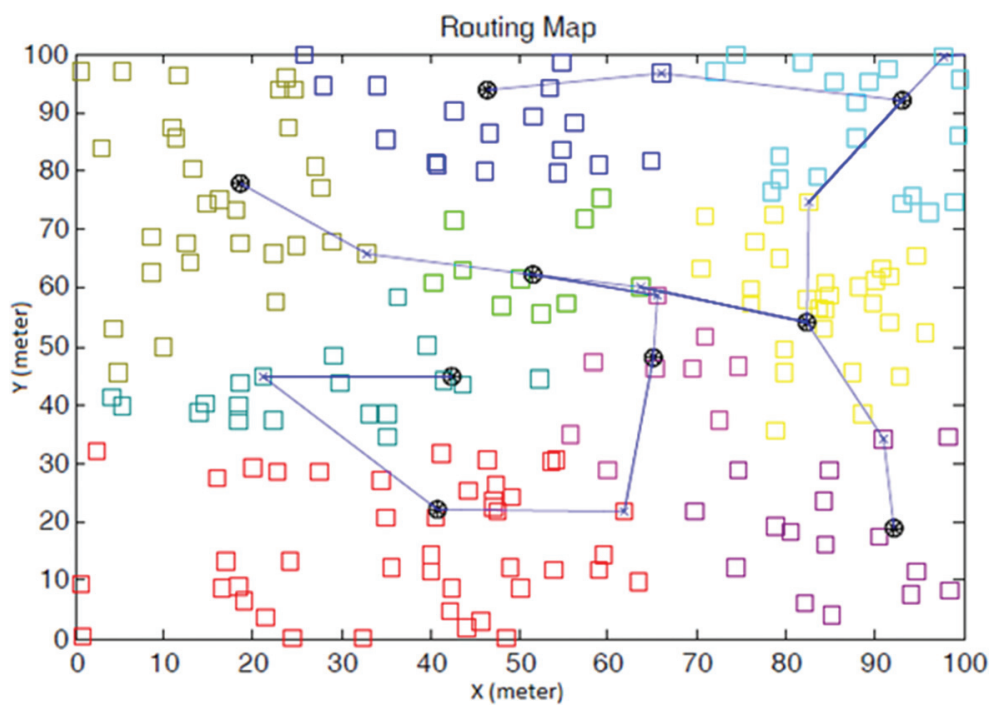

Figure 8 Routing map using LEACH. 


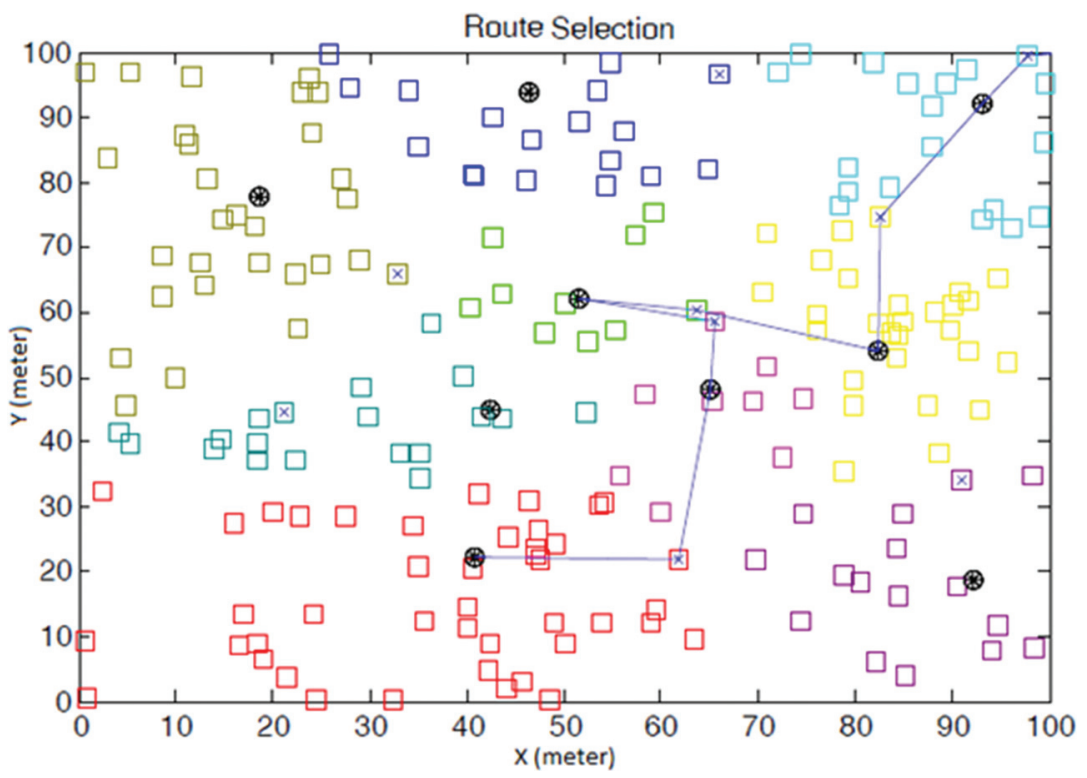

Figure 9 Optimum path selection using LEACH.

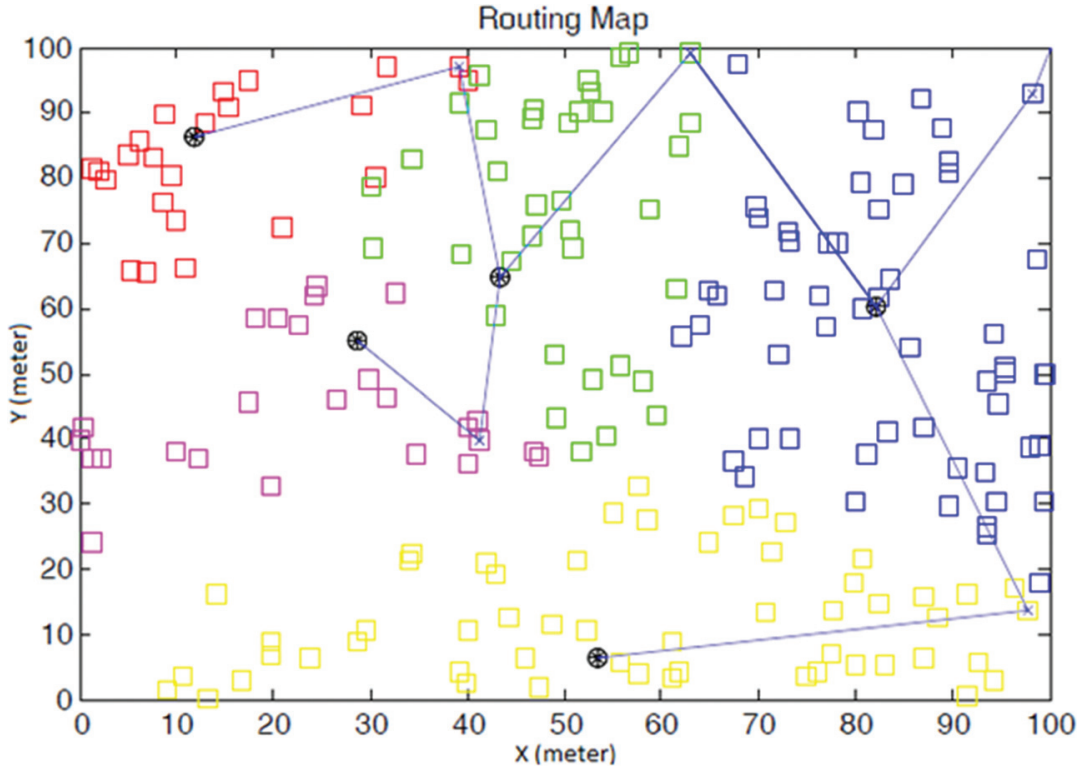

Figure 10 Routing map using the proposed algorithm. 


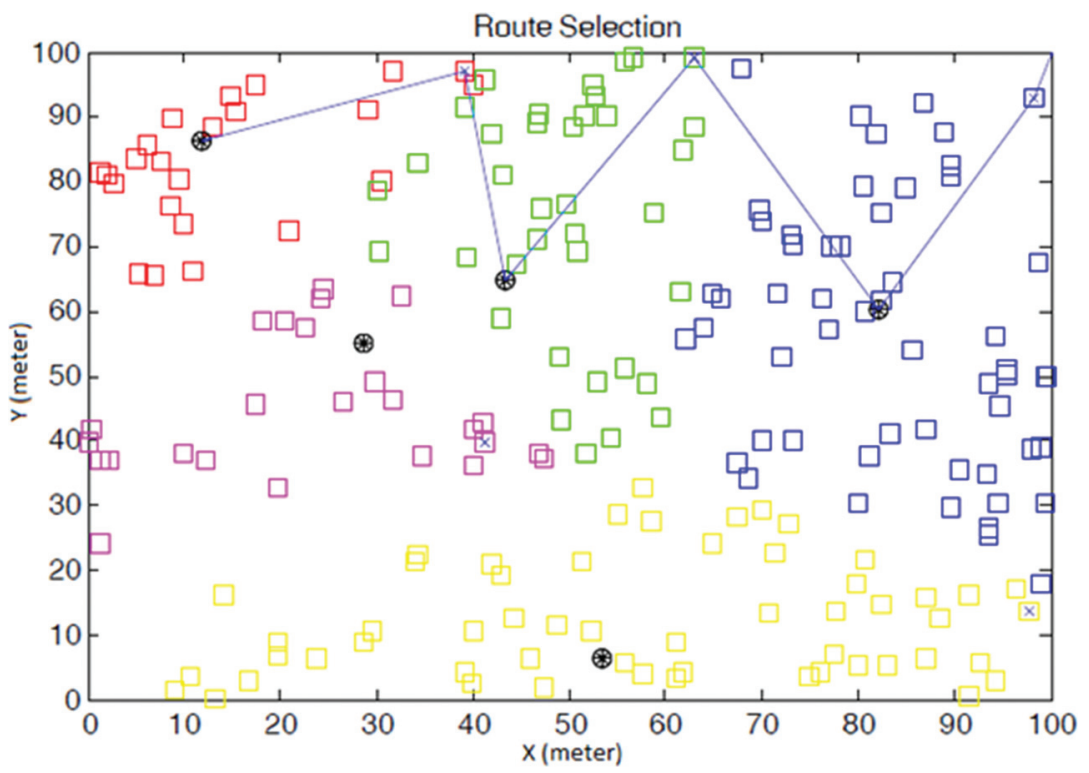

Figure 11 Optimum path selection using the proposed algorithm.

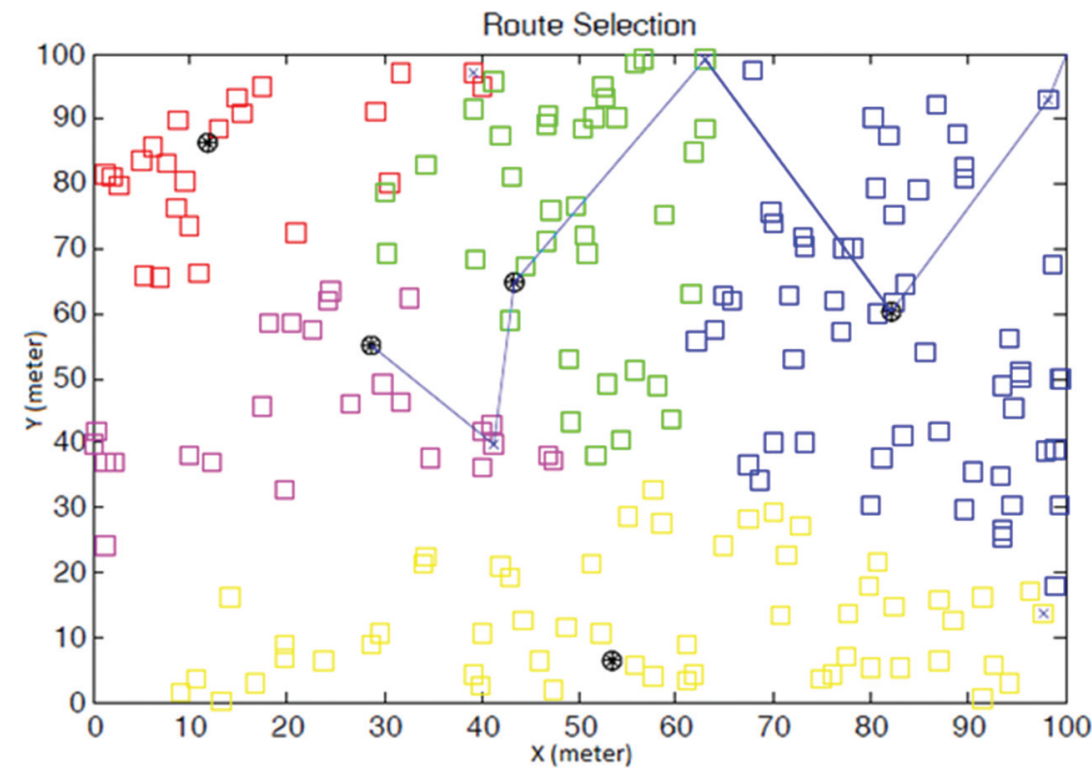

Figure 12 Routing map using Markov chain. 


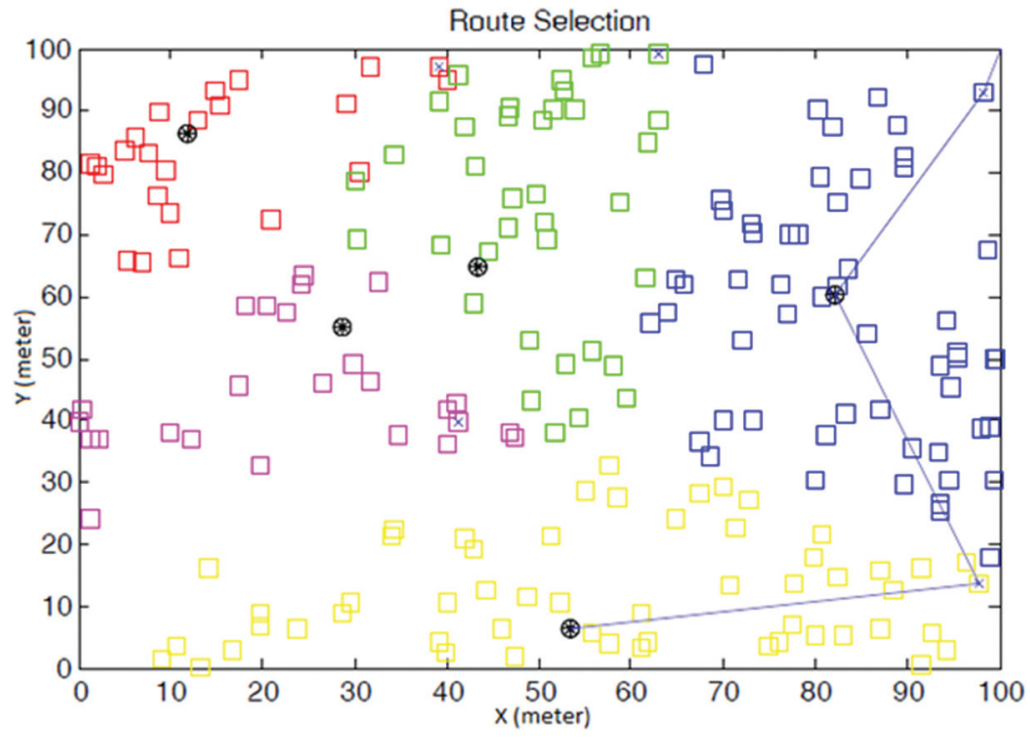

Figure 13 Optimum path selection using Markov chain.

\section{Results}

For implementations of new routing algorithms for WSNs NS-allionone2.29 simulator with Diffusion 3.2.0 code have been chosen. NS-2 is used to simulate WSNs and the new algorithms have been implemented in it. About 300 nodes distributed throughout about $400 \times 400 \mathrm{~m}^{2}$ have been used for these implementations. The $802.11 \mathrm{~b}$ protocol is used for simulation of wireless scenario Diffusion 3.2.0 in NS-allinone-2.29. The nodes have been randomly expanded in grid according to energy consumption in PCM-CIA WLAN card as NS-2. At the same time some simulations are done by using MATLAB. Simulation parameters are given in Table 1.

Table 1 Simulation parameters

\begin{tabular}{llll}
\hline Parameter & Value & Parameter & Value \\
\hline Layer 3 Protocol & Diffusion & Radio Propagation & Two-way \\
Layer 2 Protocol & IEEE 802.11g & Packet Size & 4 Kbit \\
$E_{T x-\text { elec }}$ & $50 \mathrm{~nJ} / \mathrm{bit}$ & Data Rate & $1 \mathrm{Mbps}$ \\
$E_{R x-\text { elec }}$ & $50 \mathrm{~nJ} / \mathrm{bit}$ & Radio Range & $90 \mathrm{~meter}$ \\
Sensing Power & $4 \mathrm{~nJ} / \mathrm{bit}$ & Sensing Range & $13 \sim 48$ meter \\
Area & $160 \times 160 \mathrm{~m}^{2}$ & Number of Nodes & 250 \\
\hline
\end{tabular}


Figure 14 compares the remainder energy between LEACH and the proposed algorithm. The energy of LEACH nodes diminished by the 350th time slice whereas the proposed algorithm reduced the energy consumption. Figure 15 illustrates the number of packets lost per time slice, which is lower

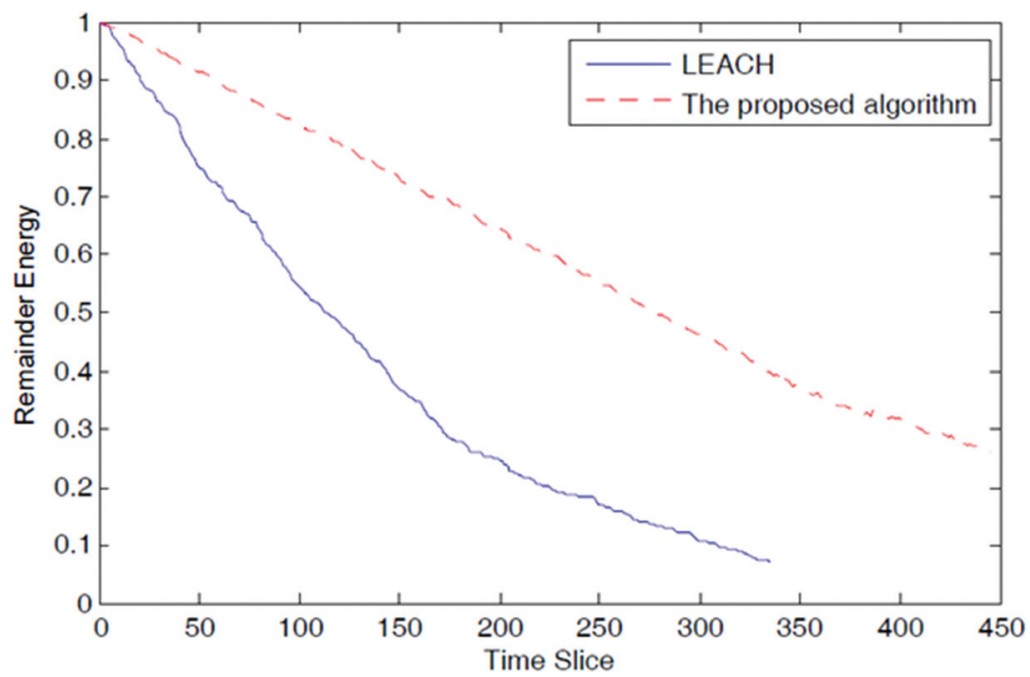

Figure 14 Energy consumption between LEACH and the proposed algorithm.

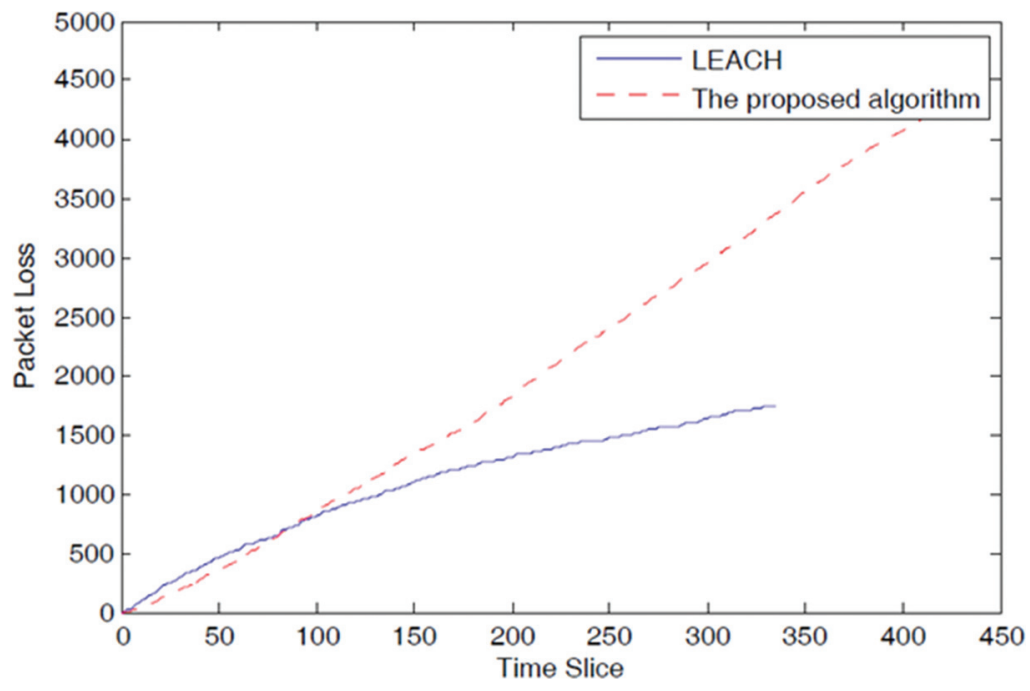

Figure 15 Packet loss between LEACH and the proposed algorithm. 
in the proposed algorithm in the first 100 time slices but experienced a steady growth, surpassing the packet loss achieved by LEACH. This is proportionally reflected in the delivery times presented in Figure 16.

Figure 17 summarizes the lifetime obtained using the proposed algorithm, highlighting a lower number of dead nodes in any given time slice as compared to LEACH.

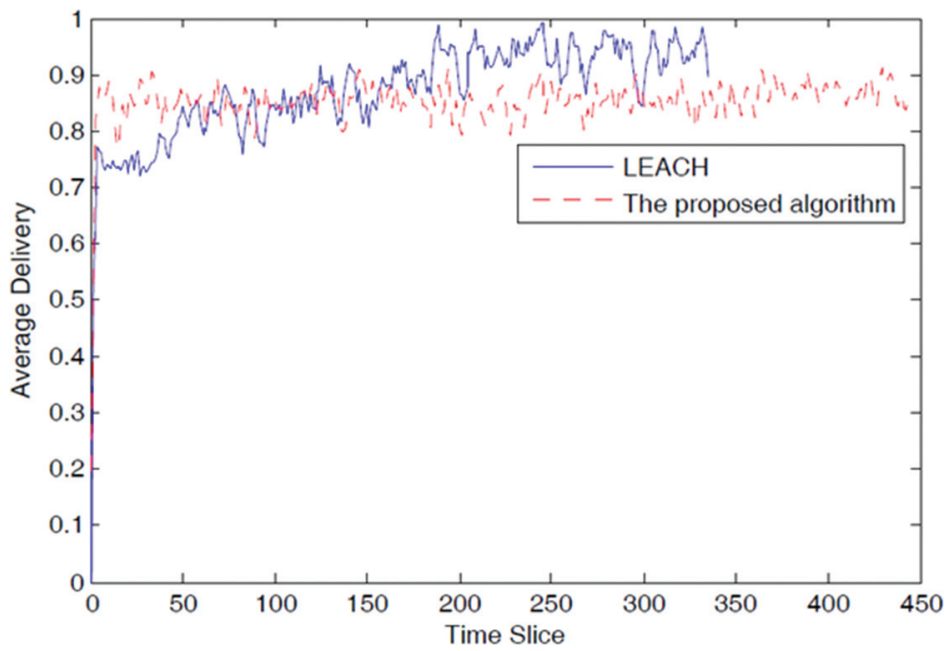

Figure 16 Delivery time between LEACH and the proposed algorithm.

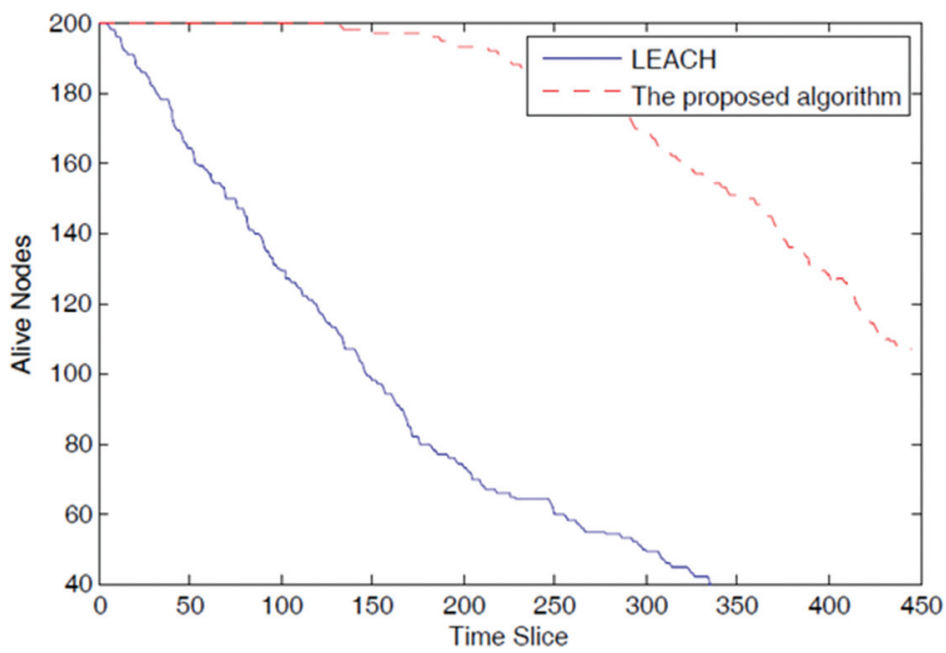

Figure 17 Live nodes between LEACH and the proposed algorithm. 


\section{Conclusion}

In this paper, we proposed a method achieving a more efficient energy utilization by sensor nodes. The simulation results demonstrate that the proposed algorithm could save energy. The method improved the network lifetime and energy consumption, at the expense of packet loss and data delivery. When the network started, this method achieves a higher data-delivery than LEACH algorithm but after the 100th time slice, LEACH performed better in terms of data delivery and packet loss. The proposed method opens up new gateways for future works to optimize energy usage by cluster heads and nodes in WSNs.

\section{Acknowledgment}

This work is supported in part by Doctoral Graduate Research Assistantship from UNLV Graduate College and in part by NSF award \#EPS-IIA-1301726 (EPSCoR NEXUS).

\section{References}

[1] Khan, N. A., Saghar, K., Ahmad, R., and Kiani, A. K. (2016). "Achieving energy efficiency through load balancing: a comparison through formal verification of two WSN routing protocols," in 13th International Bhurban Conference on Applied Sciences, and Technology (IBCAST) (Rome: IEEE), 350-354. doi: 10.1109/IBCAST.2016.7429901.

[2] Mirnabibaboli, M. (2013). The Enhancements of Network Lifetime and Supporting Mobility by Utilizing Suitable Routing in the Wireless Sensor Network. Ph.D. Dissertation, National Academy of Sciences of Armenia, Armenia.

[3] Dugaev, D., Zinov, S., Siemens, E., and Shuvalov, V. (2015). "A survey, and performance evaluation of ad-hoc multi-hop routing protocols for static outdoor networks," in International Siberian Conference on Control, and Communications (SIBCON) (Rome: IEEE), 1-11. doi: 10.1109/SIBCON.2015.7147048.

[4] Brar, G. S., Rani, S., Chopra, V., Malhotra, R. Song, H. and Ahmed, S. H. (2016). Energy efficient direction-based PDORP routing protocol for WSN. IEEE Access, 4, 3182-3194. doi: 10.1109/ACCESS.2016.2576475. 
[5] Jiang, Q., and Manivannan, D. (2004). "Routing protocols for sensor networks," in Consumer Communications and Networking Conference (CCNC) (Rome: IEEE), Las Vegas, NV, USA, 93-98. doi: 10.1109/CCNC.2004.1286839

[6] Wang, Y. H., Hsu, C. P., Lin, Y. C., Kuo, C. S., and Ho, H. Y. (2007). "A Routing Method by Reactive Energy Decision in WSNs," in 21st International Conference on Advanced Information Networking, and Applications Workshops, AINAW '07 (Rome: IEEE), 701-770. doi: 10.1109/AINAW.2007.49.

[7] Salehian, S., Masoumiyan, F., and Udzir, N. I. (2012). "Energy-efficient intrusion prediction in WSN," in International Conference on Cyber Security, Cyber Warfare, and Digital Forensic (CyberSec) (Rome: IEEE), 207-212.

[8] Intanagonwiwat, C., Govindan, R., and Estrin, D. (2000). "Directed diffusion: a scalable, and robust communication paradigm for sensor networks," in Proceedings of the 6th Annual International Conference on Mobile Computing, and Networking (MOBICOM '00) (Rome: IEEE), pp. 56-67.

[9] Jain, V., and Khan, N. A. (2014). "Simulation analysis of directed diffusion and SPIN routing protocol in wireless sensor network," in 2014 Conference on IT in Business, Industry and Government (CSIBIG), Indore, India, 2014, pp. 1-6. doi: 10.1109/CSIBIG.2014.7056990.

[10] Heinzelman, W. R., Kulik, J., and Balakrishnan, H. (1999). "Adaptive protocols for information dissemination in wireless sensor networks," in Proceedings of the 5th Annual ACM/IEEE International Conference on Mobile Computing, and Networking (MobiCom_99) (Rome: IEEE), Seattle, WA.

[11] Heinzelman, W. R., Chandrakasan, A., and Balakrishnan, H. (2000). "Energy-efficient communication protocol for wireless microsensor networks," in Proceedings of the 33rd Annual Hawaii International Conference on System Sciences (Rome: IEEE), 10. doi: 10.1109/HICSS.2000.926982.

[12] Migabo, M. E., Djouani, K., Kurien, A. M., and Olwal, T. O. (2015). A stochastic energy consumption model for wireless sensor networks using GBR techniques. AFRICON, 1-5. doi: 10.1109/AFRCON.2015.7331987.

[13] Faruque, J., and Helmy, A. (2003). Gradient-based routing in sensor networks. ACM SIGMOBILE Mobile Comput. Commun. Rev. Arch. 7, $50-52$. 
[14] Kim, D., Ha, S., and Choi, Y. (1998). "K-hop cluster-based dynamic source routing in wireless ad-hoc packet radio network," in 48th IEEE Vehicular Technology Conference, VTC 98, Vol. 1, Ottawa, ON, 224-228. doi: 10.1109/VETEC.1998.686541.

[15] Lindsey, S., and Raghavendra, C. S. (2002). "PEGASIS: Power Efficient Gathering in Sensor Information Systems," in Proceedings of IEEE Aerospace Conference (Rome: IEEE).

[16] Zonouz, A. E., Xing, L., Vokkarane, V. M., and Sun, Y. (2016). Hybrid wireless sensor networks: a reliability, cost and energy-aware approach. IET Wirel. Sensor Syst. 6, 42-48. doi: 10.1049/iet-wss.2014.0131.

[17] Mishra, A. K., R. Us. Rahman, Bharadwaj, R., and Sharma, R. (2015). "An enhancement of PEGASIS protocol with improved network lifetime for WSNs," in IEEE Power, Communication, and Information Technology Conference (PCITC), 142-147.

[18] Bushnag, A., Alessa, A., Li, M., and Elleithy, K. (2015). "Directed diffusion based on weighted Grover's quantum algorithm (DWGQ)," in Systems, Applications, and Technology Conference (LISAT), IEEE Long Island, 1-5.

[19] Chughtai, O., N. Badruddin and Awang, A. (2014). "A congestionaware and energy efficient traffic Load balancing Scheme for routing in WSNs," in TENCON 2014-2014 IEEE Region 10 Conference, Bangkok, 1-6. doi: 10.1109/TENCON.2014.7022431.

[20] Abbasi, A., and Younis, M. (2007). A survey on clustering algorithms for WSNs. Comput. Commun. 2826-2841.

[21] Gupta, G., and Younis, M. (2003). "Performance evaluation of loadbalanced clustering of wireless sensor networks," in 10th International Conference on Telecommunications ICT 2003 (Rome: IEEE), Vol. 2, 1577-1583. doi: 10.1109/ICTEL.2003.1191669.

[22] Du, X., Xiao, Y., and Dai, F. (2006). Increasing network lifetime by balancing node energy consumption in heterogeneous sensor networks. Wirel. Commun. Mobile Comput. 125-136.

[23] AlAmri, H., Abolhasan, M., Franklin, D. R., and Lipman, J. (2014). "Optimised relay selection for route discovery in reactive routing." Ad Hoc Netw., pp. 70-88.

[24] Chughtai, O., Badruddin, N., Awang, A., and Rehan, M. (2016). Congestion-aware and traffic load balancing scheme for routing in WSNs. Telecommun. Syst., 1-24. 
[25] Ren, F., He, T. Das S. K., and Lin, C. (2011). Traffic-Aware Dynamic Routing to Alleviate Congestion in Wireless Sensor Networks. IEEE Trans. Parallel Distribut. Syst. 22, 1585-1599. doi: 10.1109/TPDS.2011.24.

[26] Kinoshita, K., Inoue, N., Tanigawa, Y., and Tode, H. T. (2016). "Watanabe. Fair routing for overlapped cooperative heterogeneous WSNs." IEEE Sensors J., 3981-3988.

[27] Marques, B., and Ricardo, M. (2016). Energy-efficient node selection in application-driven WSN. Wirel. Netw., 1-30.

[28] Benyamina, D., Hafid, A., Hallam, N., and Gendreau, M. (2012). A hybrid nature-inspired optimizer for wireless mesh networks design. Comput. Commun. 35, 1231-1246.

[29] Abdelhakim, M., Liang, Y., and Li, T. (2016). Mobile coordinated wireless sensor network: an energy efficient scheme for realtime transmissions. IEEE J. Select. Areas Commun. 34, 1663-1675. doi: 10.1109/JSAC.2016.2545383.

[30] Ren, J. Zhang, Y., Zhang, K., Liu, A., Chen J., and Shen, X. S. (2016). Lifetime and energy hole evolution analysis in data-gathering wireless sensor networks. IEEE Trans. Ind. Inform. 12, 788-800. doi: 10.1109/TII.2015.2411231.

[31] Deva Sarma, H. K., Mall, R., and Kar, A. (2016). E2R2: Energy-efficient and reliable routing for mobile wireless sensor networks. IEEE Syst. J. 10, 604-616. doi: 10.1109/JSYST.2015.2410592.

\section{Biographies}

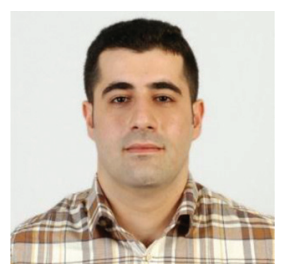

S. Tayeb received the M.S. degree (Magna Cum Laude) in radio engineering and communications and the B.S. degree (Magna Cum Laude) in telecommunications engineering from the State Engineering University of Armenia in 2012 and 2010, respectively. He is currently a Ph.D. candidate in the department of electrical and computer engineering at University of Nevada Las Vegas (UNLV). He holds CCIE R\&S, CCDP, CCNP R\&S, and CCAI 
from Cisco; CNSS 4011 Recognition from NSA; TKT from Cambridge; and VMCA-DCV from VMware. Prior to joining UNLV, he was an instructor and instructor trainer at Cisco Networking Academy where he was recognized as top $\% 5$ expert level instructors globally. He has been invited to deliver instructor-level courses in various countries around Europe, Middle East, Africa, and North America. He has authored/co-authored several research papers on network security, Wireless Sensors Networks, Internet of Things, and Big Data. His research interests span the areas of Internet of Things, Information Assurance, Security, and Wireless Sensor Networks utilizing such tools as Deep Learning and Big Data Analytics. He is a member of IEEE, ISOC, Teachers without Borders, and NSPE.

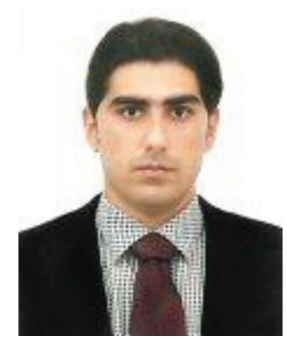

M. Mirnabibaboli was born in Babol, Iran, in 1984. He received the B.S. degree in Software Engineering from the University of Sari in 2007. His fields in M.S. and Ph.D. were Information Technology-Network Engineering from IT department of QIUZ and NASRA. He taught Computer courses for B.S. and M.S. students at universities of IAU, PNU, UMZ, and UAST in Iran between 2009 and 2015. He was adviser and member of Informatics Councils of IT in AgriBank of Iran in 2014 and 2015.

In 2016, he joined the Department of Electrical and Computer Engineering at University of Nevada, Las Vegas, as a PostDoc-Research Scholar. He researched on WSNs, Data Mining, Bio-Data Mining and Cloud Computing in the past 8 years. 


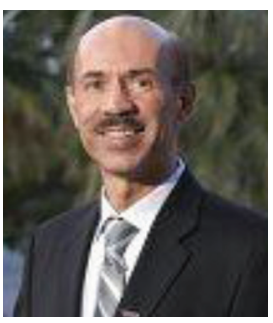

S. Latifi, an IEEE Fellow, received the Master of Science degree in Electrical Engineering from Fanni, Teheran University, Iran in 1980. He received the Master of Science and the Ph.D. degrees both in Electrical and Computer Engineering from Louisiana State University, Baton Rouge, in 1986 and 1989, respectively. He is currently a Professor of Electrical Engineering at the University of Nevada, Las Vegas. Dr. Latifi is the co-director of the Center for Information Technology and Algorithms (CITA) at UNLV. He has designed and taught undergraduate and graduate courses in the broad spectrum of Computer Science and Engineering in the past three decades. He has given seminars on cyber-related topics all over the world. He has authored over 250 technical articles in the areas of networking, cybersecurity, image processing, biosurveillance, biometrics, document analysis, fault tolerant computing, parallel processing, and data compression. His research has been funded by NSF, NASA, DOE, DoD, Boeing, Lockheed and Cray Inc. Dr. Latifi was an Associate Editor of the IEEE Transactions on Computers (1999-2006), an IEEE Distinguished Speaker (1997-2000), and Co-founder and General Chair of the IEEE Int'l Conf. on Information Technology (2004-2015). Dr. Latifi is the recipient of several research awards, the most recent being the Silver State Research Award (2014). He is also a Registered Professional Engineer in the State of Nevada. 